\title{
Vasodilator Stress Impairs the Left Ventricular Function Obtained With Gated Single-Photon Emission Computed Tomography in Patients With Known or Suspected Coronary Artery Disease
}

\author{
Keiichi Odagiri, MD, PhD; Akihiko Uehara, MD, PhD; Chinori Kurata, MD, PhD
}

(Circ J 2010; 74: 2666-2673)

The authors apologize for the omission of co-authors' names.

Incorrect:

Keiichi Odagiri, MD, PhD; Akihiko Uehara, MD, PhD; Chinori Kurata, MD, PhD

Division of Cardiology, Internal Medicine III, Hamamatsu University School of Medicine, Hamamatsu (K.O.); Yamaha Health Care Center, Hamamatsu (A.U., C.K.), Japan

Correct:

Keiichi Odagiri, MD, PhD; Masashi Machii, MD; Takamitsu Tanaka, MD; Akihiko Uehara, MD, PhD; Chinori Kurata, MD, PhD; Harumi Sakahara, MD, PhD; Yasuo Takehara, MD, PhD; Masao Saotome, MD, PhD; Tsuyoshi Urushida, MD; Hideki Katoh, MD, PhD; Hiroshi Satoh, MD, PhD; Hideharu Hayashi, $\mathrm{MD}, \mathrm{PhD}$

Division of Cardiology, Internal Medicine III (K.O., M.M., T.T., M.S., T.U., H.K., H. Satoh, H.H.), Department of Radiology (H. Sakahara, Y.T.), Hamamatsu University School of Medicine, Hamamatsu; Yamaha Health Care Center, Hamamatsu (A.U., C.K.), Japan 